\title{
Optimization Based High-Speed Railway Train Rescheduling with Speed Restriction
}

\author{
Li Wang, ${ }^{1,2}$ Wenting Mo, ${ }^{3}$ Yong Qin, ${ }^{1}$ Fei Dou, ${ }^{2}$ and Limin Jia ${ }^{1}$ \\ ${ }^{1}$ State Key Laboratory of Rail Traffic Control and Safety, Beijing Jiaotong University, Beijing 100044, China \\ ${ }^{2}$ School of Traffic and Transportation, Beijing Jiaotong University, Beijing 100044, China \\ ${ }^{3}$ IBM China Research Lab, 2F Diamond A, Zhong Guan Cun Software Park, Beijing 100193, China
}

Correspondence should be addressed to Limin Jia; jialm@vip.sina.com

Received 16 August 2013; Revised 17 October 2013; Accepted 6 November 2013; Published 12 January 2014

Academic Editor: Wuhong Wang

Copyright (C) $2014 \mathrm{Li}$ Wang et al. This is an open access article distributed under the Creative Commons Attribution License, which permits unrestricted use, distribution, and reproduction in any medium, provided the original work is properly cited.

\begin{abstract}
A decision support framework with four components is proposed for high-speed railway timetable rescheduling in case of speed restriction. The first module provides the speed restriction information. The capacity evaluation module is used to evaluate whether the capacity can fulfill the demand before rescheduling timetable based on deduction factor method. The bilayer rescheduling module is the key of the decision support framework. In the bilayer rescheduling module, the upper-layer objective is to make an optimal rerouting plan with selected rerouting actions. Given a specific rerouting plan, the lower-layer focuses on minimizing the total delay as well as the number of seriously impacted trains. The result assessment module is designed to invoke the rescheduling model iteratively with different settings. There are three prominent features of the framework, such as realized interaction with dispatchers, emphasized passengers' satisfaction, and reduced computation complexity with a bilayer modeling approach. The proposed rescheduling model is simulated on the busiest part of Beijing to Shanghai high-speed railway in China. The case study shows the significance of rerouting strategy and utilization of the railway network capacity in case of speed restriction.
\end{abstract}

\section{Introduction}

China is extensively developing the infrastructure of highspeed railway. The target is to cover its major economic areas with a high-speed railway network, which consists of four horizontal and four vertical lines, in the following several years. The network scale is much larger than any other existing ones in the world. In the network, BeijingShanghai high-speed line connects Beijing (the capital of China) and Shanghai (the biggest economic centre of China), and it goes through Yangtze River Delta region (the best developed area in China). Thus, people describe it as the North-South Aorta of China. As designed, trains of different speeds will be operated in a mixed way on the network with a minimum headway of 3 minutes. In other words, it has the characteristics of high train speed, high train frequency, and mixed train speed (HHM).

A basic train timetable essentially provides the arrival time of trains at stations and the corresponding departure time from the stations. Based on the timetable, the dwell time of trains at the stations (which is 0 if they do not stop) and the departure order of trains from the trains can be derived. During daily operation, disturbances to the timetable are inevitable, which may be caused by emergencies like natural disasters, accidents, or maintenance requests. In case of the disturbance, dispatchers must adopt proper emergency management strategies to ensure operation safety. Consequently, they need to reschedule the timetable to avoid conflict and recover to the original timetable. Drivers will then adjust the train speed and dwell time according to the new arrival and departure time given in the rescheduled timetable.

In the context of transportation operation, the timetable rescheduling problem is famous of its NP hardness. What is worse, it is very hard to find a generalized rescheduling algorithm suitable for all kinds of emergency cases because of various railway infrastructure and various emergency management actions. Except adjusting arrival/departure time, all the actions taken for rescheduling are called rerouting actions 
in this paper, including cancelling trains, merging trains, and making a detour.

Setting speed restriction is one of the most common strategies used by dispatchers facing emergency cases. With fixed departure order of trains at all the stations and unchanged time interval between consecutive departures, the throughput of a railway section over a period of time will decrease in case of speed restriction. In other words, trains may be delayed or even cancelled. The impacted number of trains is decided by the size of the restricted section, the time period in restriction, and the restricted speed. On June 20, 2010, the southern part of the existing BeijingShanghai line experienced speed restriction for heavy rain storm. As announced, 18 trains departing from Shanghai were cancelled, thereby more than 20,000 passengers being affected. Also, the rest in-service trains were more or less delayed. Considering the high train speed and high train frequency of high-speed lines, the impact of speed restriction would be more serious than on existing lines (in this paper, the existing nonhigh speed railway line is called existing line for short). On the other hand, high-speed lines are more passenger-oriented than existing lines, where punctuality is extraordinarily important. Thus, it is significant to build a responsive and effective decision support mechanism to handle the timetable rescheduling in case of speed restriction for high-speed lines. However, there are two challenges to achieve this goal. One is the interaction between the rescheduling algorithm and the dispatchers. Since the dispatchers have rich experience, their advice and decision on the rescheduling strategies are highly valuable. The other one is the HHM characteristics of the high-speed network.

In this paper, we investigate the design and implementation of a decision support mechanism for timetable rescheduling in case of speed restriction as mentioned above. Note that the three prominent features of this mechanism are realized interaction with dispatchers, emphasized passengers' satisfaction, and reduced computation complexity with a bilayer modeling approach. In the rest of the paper, related research work is discussed in the section of The Literature Review. The next section describes the decision support mechanism in detail, including the mathematical modeling. A case study on the busiest part of Beijing-Shanghai highspeed line is given in the section of Case Study. The last section concludes the whole paper and gives directions for future research.

\section{The Literature Review}

The timetable rescheduling problem has been widely investigated by researchers. Some related works are reviewed, although they are quite different from our work from model creation to solving method.

Some researchers take the rerouting, cancellation, and other complicated strategies into consideration. Reference [1] developed a detailed model based on the identification of possible route conflicts with high accuracy, using the blocking time theory, when the objective is to minimize additional running times. In presence of disturbances, an algorithm detects the infeasible train routes and solves each conflict locally based on train priorities. Reference [2] regarded the train rescheduling problem as a constraint optimization problem, using passengers' dissatisfaction as the objective criterion. Then an efficient algorithm combining PERT and metaheuristics has been addressed. Experiments show that it works quite fast and it supports versatile methods of rescheduling including cancellation, change of train-set operation schedule, and change of tracks. D'Ariano et al. have developed a real-time dispatching system, called ROMA (railway traffic optimization by means of alternative graphs), to automatically recover disturbances. ROMA is able to automatically control traffic, evaluating the detailed effects of train [3] and local rerouting actions [4], while taking into account minimum distance headways between consecutive trains and the corresponding variability of train dynamics [5-7]. In order to handle large time horizons within a linear increase of computation time, the temporal decomposition approaches which decompose a long time horizon into tractable intervals to be solved in cascade with the objective of improving punctuality have been proposed in [8].

To speed up trains rescheduling computation time, some decomposition or multilayer methods are adopted. Reference [8] proposed the temporal decomposition approach that decompose a long time horizon into tractable intervals. Reference [9] proposed a bilayer optimization model within a simulation framework to deal with the high-speed railway (HSR) line planning problem. It can reduce computation complexity, and an optimal set of stop-schedules can always be generated with less calculation time. Reference [10] presented an optimization method for fast construction of time tables which can be used for dispatching or long term operation planning. This method contains two steps: constructing and iterative improving. Constructing step uses branchand-bound method to construct the first solution taking into account only restricted amount of possible decisions. Improving step adopts the genetic algorithm as an iterative improving method. These several levels of optimization method can help reduce the calculation effort.

Furthermore, [11, 12] addressed the problem of solving conflicts in railway traffic due to disturbances. The problem is formulated as a problem of rescheduling meets and overtakes of trains and has been dealt with in a two-level process. The upper level handles the order of meets and overtakes of trains on the track sections while the lower level determines the start and end times for each train and the sections it will occupy. Reference [13] dealt with analyzing dispatchers' decision process in intertrain conflict resolutions and developing a heuristic algorithm for rescheduling trains by modifying existing meet/pass plans in conflicting situations in a singletrack railway. A system's approach is used in construction of the heuristic algorithm, which is based on inter-train conflict management. Reference [14] introduced an optimization model based on improved symmetric tolerance approach, which could reschedule the timetable timely due to unexpected interference with little cost and risk. Reference [15] investigated the train re-scheduling problem by optimization and simulation in order to obtain an exact or approximate solution. The model aims at maximizing the number of passengers transported and is solved by heuristic procedure 
based on backtracking algorithm. The model and DSS are implemented in Asturias of the Spanish National Railway Company in 1998. Reference [16] proposed a model and a solution method for the dispatching of trains on a singletrack line. Their model mainly addresses the operational problem of dispatching trains in real time but can also serve at the strategic level to evaluate the impacts of timetable or infrastructure changes on train arrival times and train delays. The formulation is a nonlinear mixed integer program that incorporates lower and upper limits on train velocities for each train on each segment. The objective function only seeks to minimize a combination of total train tardiness and fuel consumption. Based on the driver's safety approaching behavior and pedestrian safety crossing behavior, Wang et al. [17] and Guo et al. [18] proposed an intelligent driving shaping model with multiruled decision-making mechanism in the urban traffic environment.

Despite the effort devoted to solve the sophisticated rescheduling problem, neither of them proposes the closed loop bilayer feedback approach. This approach is able to simulate the scheduling process of dispatchers. Also it permits the dispatchers adding their experiences into the rescheduling process so as to improve the usability of the realtime rescheduling.

\section{Decision Support Mechanism (DSM) for Timetable Rescheduling}

Note that the railway lines considered in this paper are all double-track passenger lines with automatic blocking.

In order to handle daily operation tasks, there is usually a train operation system for dispatchers. In emergencies, dispatchers issue action commands through this system to signal system. There are 4 components in the proposed DSM as shown in Figure 1.

The speed restriction action as the first part is the input coming from the train operation system. It contains the following information.

(i) Restricted section is a part of the line where speed of trains is restricted.

(ii) Restricted period is the start and end times of a speed restriction.

(iii) Applied train type is different trains may be restricted to different speeds according to their type.

(iv) Speed limitation is the maximum speed a train that can run at.

Given the speed restriction information, the line capacity evaluation module is used to evaluate whether the capacity can fulfill all the trains scheduled in the original timetable in limited service hour. Then, the speed and capacity information are transferred to the third module. In the bilayer rescheduling model, the upper-layer objective is to make an optimal rerouting plan with selected rerouting actions. Given a specific rerouting plan, the second layer focuses on minimizing the total delay as well as the number of seriously impacted trains. Thereafter, if the rescheduling result is acceptable, the new timetable generated from the DSM will be fed back into the train operation system. Otherwise, the bilayer rescheduling will be executed in a loop till the new timetable is acceptable, so that it can be disseminated to drivers.

3.1. Line Capacity Evaluation. As mentioned in the Introduction section, the line throughput may drop dramatically during the restricted period. As a result, it may not be able to fulfill all the trains scheduled in the original timetable in limited service hour. If the capacity was not enough for the demand, the result of timetable rescheduling would be meaningless. Thus, a module is used to evaluate whether the capacity can fulfill the demand before rescheduling timetable. With the evaluation result, the dispatcher can make a decision on train canceling according to rules and their experience.

During the past several years, analytical [19-21] and simulation methods [22] are used to calculate the capacity of railway lines (either single-track or double-track). Among those methods, [23] is widely used in Europe, which can be categorized as a simulation method. It stated that railway capacity depends on both infrastructure and the timetable. Therefore, the capacity calculation according to UIC 406 requires an actual timetable. Some timetabling softwares such as RailSys have already implemented such calculation methods. However, this approach may cost long computation time and may not obtain a feasible timetable.

In this paper, we use deduction factor method [24], which is popular in recent years in China railway, to calculate the line capacity. The deduction factor method is an analytical method for calculating the capacity of high-speed rail line with various types of trains. This method considers the capacity occupancy of different trains and normalizes them into one kind of trains in order to obtain the theoretical value of capacity.

If there is only one kind of trains without stopovers on high-speed rail line, capacity of high-speed rail line with high-speed trains without stopovers can be obtained by

$$
n_{\max }=\frac{1440-t_{\text {maintenance }}-t_{\text {inefficacy }}}{I},
$$

where 1440 is the total number of minutes in a day and $t_{\text {maintenance }}$ denotes the maintenance window time. In this paper, $t_{\text {inefficacy }}$ is the capacity, which cannot be used. For instance, we have to ensure that all the trains arrive to destination before the maintenance operation starts. Hence, given the speed of trains, the last train must depart from the first station before a specific time, after which we cannot send more trains. In other words, some capacity is wasted. This is the concept behind the calculation of capacity inefficacy. In addition, $I$ is the headway between consecutive trains. This parameter is the so-called safety distance, which is determined by the number of blocks between each consecutive train. So it can be obtained by train speed and blocking distance.

On the contrary, if there are various trains with different speed on the rail line, (1) cannot precisely obtain the line capacity, as different kinds of trains may take up different 


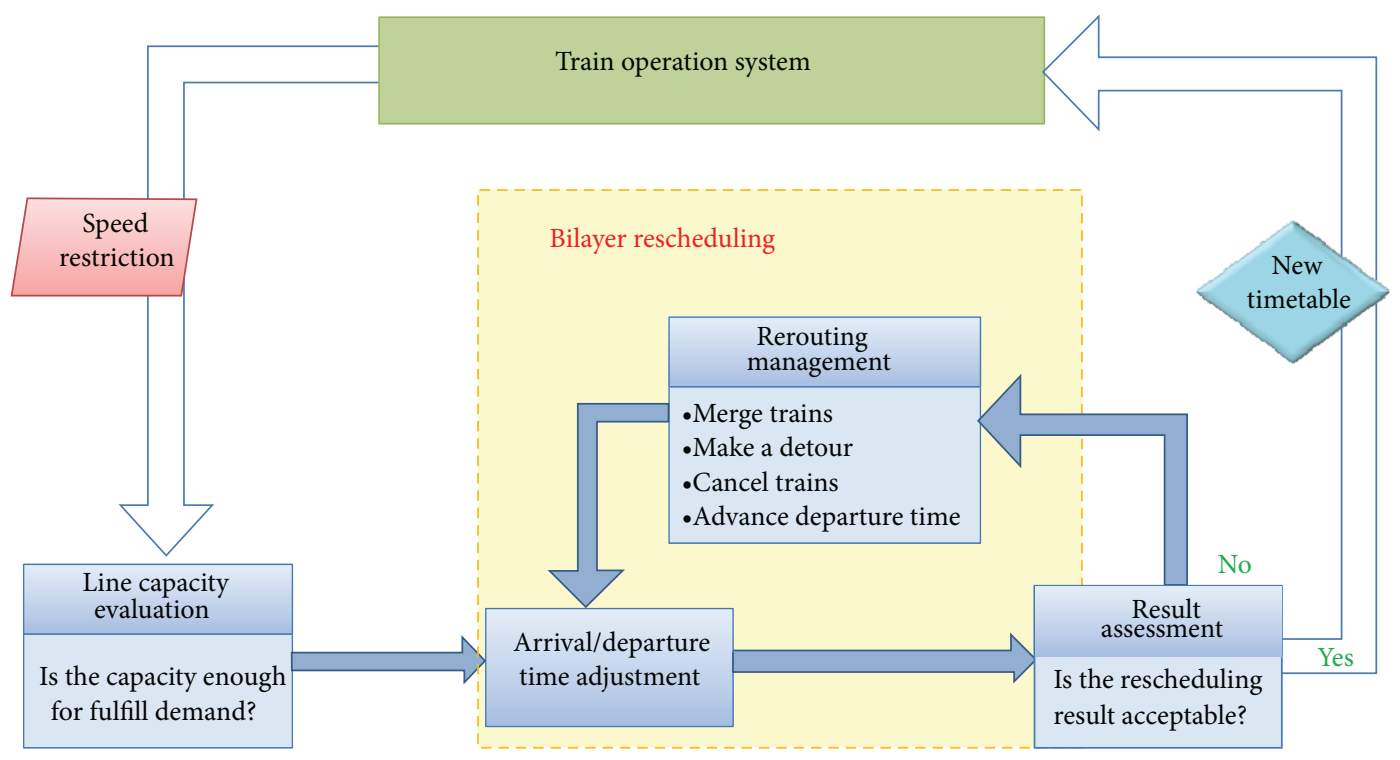

FIgURE 1: Architecture of the decision support mechanism.

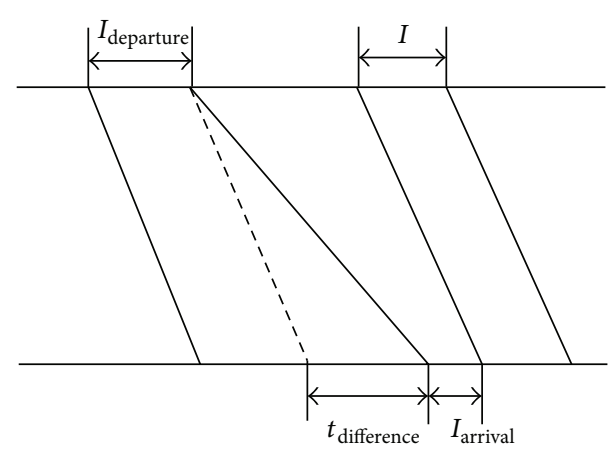

FIGURE 2: Deduction factor for single-quasihigh speed trains without stopovers with regard to high-speed trains without stopovers.

capacity. For example, as shown in Figure 2, quasihigh-speed trains will take up more "area" in the timetable. The deduction factor for single quasihigh-speed trains without stopovers with regard to high-speed trains without stopovers can be obtained by

$$
\varepsilon_{\text {high }}^{\text {nostop,quasi }}=\frac{I_{\text {departure }}+t_{\text {diffenence }}+I_{\text {arrival }}-I}{I} .
$$

Different deduction factors need to be calculated according to train operation mode, which are beyond the scope of this paper. After obtaining all kinds of deduction factors, average deduction factor can be obtained according to the ratio of different situations. Then the normalized line capacity can be calculated.

In China, there are two types of trains on most high-speed lines (high-speed trains and quasi-high-speed trains). In this situation, two kinds of deduction factors are used for capacity calculation. One arises from high-speed trains with stopovers with regard to high-speed trains without stopovers. The other one arises from the speed difference of two types of trains. A detailed example will be given in the Case Study section.

3.2. Bilayer Rescheduling. As shown in Figure 1, the rescheduling consists of three components that are executed in a loop. From the optimization perspective, the rescheduling problem is decomposed into two layers. The first layer decides the rerouted trains and the second layer adjusts the arrival and departure times of the rest of the trains. The detailed descriptions of the components are given below.

3.3. Rerouting Management. The first step in rescheduling is to decide the rerouting actions to avoid extensive delay resulted from decreased capacity.

If the capacity is not enough, it will be necessary to reduce the demand. In this paper, the following rerouting actions are considered for capacity releasing.

(i) Merging trains means to merge two trains into one, subject to two constraints. First, only trains of certain type can be merged to prevent the merged train from exceeding the maximum train length. Second, train $A$ and train $B$ can be merged only if $S S_{A} \subseteq S S_{B}$ or $S S_{B} \subseteq S S_{A}$, where $S S_{A}$ and $S S_{B}$ denote the stopping station set of $A$ and $B$, respectively. Although one train number will disappear after merging, the passengers who bought the tickets of the corresponding train will be noticed to get onboard to the merged train and are guaranteed to be able to get off at their destinations by the second merging constraint. Thus, the impact on the passengers of this rerouting action may be ignored.

(ii) Making a detour means to drive trains off the highspeed line and use the intercity line or existing line when residual capacity is available. 
(iii) Cancelling means to cancel trains. When a train is cancelled, not only the tickets must be refunded but also the reputation would be damaged. So, this rerouting action is of the lowest priority.

Note that the calculated capacity is the upper bound of the real capacity. Theoretically, if the capacity is larger than the demand, all the trains can travel from the origin to the destination in the service hour. Nonetheless, the capacity may not be fully utilized due to sparse departure from the origin station. Thus, advancing departure time is another rerouting action, which can be taken when the capacity is not fully utilized.

The optimization at this layer aims at finding an optimal rerouting plan with the minimum cost. The cost is associated with the impacts of the above rerouting actions on passengers, including the refundable paid by railway company and the extra transfer taken by passengers. This reflects the passengeroriented principle in the high-speed railway.

3.4. Arrival/Departure Time Adjustment. Given the rerouting plan, the trains remaining to run on the high-speed line are known. Thereafter, the optimization objective is to minimize the weighted delay of these trains, where the weights correspond to their priorities. The objective can be achieved by adjusting the arrival/departure time to/from stations. In practice, the speed of trains running between two consecutive stations, the dwell time of trains at stations, and the departure order of trains from stations may need to be changed according to the arrival/departure time adjustment.

The advantages of bilayer decomposition can be interpreted from different angles. From the system perspective, it facilitates the interaction with dispatchers. They are enabled to explicitly control the rerouting actions. From the problem solving perspective, it effectively reduces the complexity of optimization. Long computation time is usually a curse for rescheduling problems because of the large number of decision variables, which prevents the application of optimization-based automatic timetable rescheduling algorithms being used in real world.

3.5. Decision Tree-Based Result Assessment. A decision tree is mined from an evaluation rule set for rescheduling result assessment purpose. A rule defines the conditions for accepting or rejecting a rescheduled timetable. The rule set is generated from the dispatchers' experience on the acceptability of a rescheduled timetable. Basically, extensive delay and long delay of single trains are not acceptable to passengers in high-speed lines. Table 1 gives an example of the evaluation rules, where $\theta_{1}=30 \mathrm{mins}$ and $\theta_{2}=60 \mathrm{mins}$.

Overlapping or redundancy may exist in the rule set, as the rules are generated from experience. Greedy algorithm can be used to search for the smallest decision tree based on a given rule set [23]. In a decision tree, each node is a test for an attribute, each branch corresponds to test results, and each leaf assigns a decision. For illustration purpose, Figure 3 shows the smallest decision tree obtained from the rules given in Table 1.

\section{Mathematical Modeling of the Bilayer Rescheduling}

Mixed integer linear programming (MILP) is used to model the proposed bilayer rescheduling.

4.1. Input Data. The numerical inputs are described as follows:

inis $_{p}$, inie ${ }_{p}$ : initial start and end times of interval $p$ according to original timetable;

$z_{p}$ : the minimum size of interval $p$. If $p$ is the possession of a station, $d_{p}$ stands for the minimum dwell time of the train associated with $p$. Otherwise, it stands for the minimum running time of the train;

$f_{k}$ : the minimum separation time of two intervals on station $k$, that is, end time of an interval and start time of the subsequent interval;

$h w_{k}$ : the headway of section $k$, that is, start time of an interval and start time of the subsequent interval;

$g_{i}$ : the length of train $i$. Only if $g_{i}=G$, train $i$ can be merged. $G$ is a constant input;

$c_{i}^{\text {cancel }}, c_{i}^{\text {detour }}$, and $c_{i j}^{\text {merge }}$ : the cost of canceling train $i$, detouring train $i$, and merging train $i$ to train $j$, respectively;

$c_{i}^{\text {delay }}$ : the cost per time unit delay for train $I$;

$w_{i}$ : delay tolerance for train $I$;

$$
h_{p} \begin{cases}1, & \text { if interval } p \text { is a planned stop } \\ & \text { where } p \in \text { Itv } \\ 0, & \text { otherwise; }\end{cases}
$$

$\Delta C$ : the total residual capacity of alternative lines;

$\delta$ : the minimal number of trains to be applying rerouting actions, which is obtained from line capacity evaluation module;

M: a very big integer, for example, 100000.

Some sets are defined in Table 2 for modeling. Note that the segment between two stations is called "section", while the station segment is called "station" for short.

\subsection{Model of Rerouting Management Layer}

\subsubsection{Decision Variables}

$$
\begin{gathered}
q_{i}= \begin{cases}1, & \text { if train } i \text { is canceled, where } i \in \operatorname{Trn} \\
0, & \text { otherwise, }\end{cases} \\
m_{i j}= \begin{cases}1, & \text { if train } i \text { is merged to train } j, \\
0, & \text { where } i, j \in \operatorname{Trn}, g_{i}=g_{j}=G\end{cases}
\end{gathered}
$$


TABLE 1: Example of evaluation rules.

\begin{tabular}{lccc}
\hline $\begin{array}{l}\text { Percentage of trains being delayed } \\
\text { more than } \theta_{1}\end{array}$ & $\begin{array}{c}\text { Percentage of trains being delayed } \\
\text { more than } \theta_{2}\end{array}$ & $\begin{array}{c}\text { Number of delayed } \\
\text { highpriority trains }\end{array}$ & Acceptable (outcome) \\
\hline$\leq 80 \%$ & $\geq 50 \%$ & -2 & No \\
$\leq 20 \%$ & - & $\leq 15$ & Yes \\
$\leq 50 \%$ & - & $\leq 15$ & Yes \\
- & & & Yes \\
$\vdots$ & & & \\
\hline
\end{tabular}

TABLE 2: Sets defined for modeling.

\begin{tabular}{|c|c|c|c|}
\hline Set name & Description & Size & $\begin{array}{l}\text { Index } \\
\text { of set }\end{array}$ \\
\hline $\operatorname{Trn}$ & All the trains & $T$ & $i$ \\
\hline Seg & All the segments & $S$ & $k$ \\
\hline $\operatorname{Stn} \subseteq$ Seg & All the stations & $N$ & - \\
\hline $\operatorname{Sec} \subseteq \operatorname{Seg}$ & All the sections & $S-N$ & - \\
\hline Itv & $\begin{array}{l}\text { All the intervals where an interval is the possession of a segment by a train with specified start and end } \\
\text { times }\end{array}$ & - & $p$ \\
\hline $\operatorname{Itv}_{i} \subseteq \mathrm{Itv}$ & The ordered set of intervals for train $i$ according to the original timetable & - & - \\
\hline $\operatorname{Itv}_{i}^{\operatorname{stn}} \subseteq \operatorname{Itv}_{i}$ & The ordered set of intervals associated with stopping at stations for trains $i$ & - & - \\
\hline $\operatorname{Itv}_{k} \subseteq \mathrm{Itv}$ & The ordered set of intervals for segment $k$ according to the original timetable & - & - \\
\hline $\operatorname{Trk}_{k}$ & All the tracks of station $k$ & $L_{k}$ & $l$ \\
\hline
\end{tabular}

Remarks: (1) for the ease of understanding, we use $p$ as the index for every interval-related set (i.e., $\operatorname{Itv}_{\text {, }} \operatorname{Itv}_{i}$, $\operatorname{Itv}_{i}^{\text {stn }}$, and $\operatorname{Itv}_{k}$ ). In each of the interval-related sets, $p+1$ indicates the first subsequent interval of $p$, and $\tilde{p}$ indicates all the subsequent intervals of $p$.

Note that if train $i$ is merged to train $j$, the train number of $i$ will no longer exist:

$$
o_{\mathrm{i}}= \begin{cases}1, & \text { if train } i \text { makes a detour with existing } \\ \text { line, where } i \in \operatorname{Trn}\end{cases}
$$

\subsubsection{Objective Functions}

(i) To minimize the rerouting cost,

$\operatorname{Minimize} \sum_{i \in \operatorname{Trn}}\left(c_{i}^{\text {cancel }} q_{i}+c_{i}^{\text {detour }} o_{i}+c_{i j}^{\text {merge }} \sum_{j \in \operatorname{Trn}} m_{i j}\right)$.

\subsubsection{Constraints}

(ii) Cancelled trains cannot make detour.

$$
q_{i}+o_{i} \leq 1, \quad i \in \operatorname{Trn} .
$$

(iii) If a train is eligible to be merged, it can be merged to (with) at most one other train. The merged train cannot be cancelled:

$$
\begin{gathered}
\sum_{i=j} m_{i j}=0, \quad i, j \in \operatorname{Trn}, \\
\sum_{j \in \operatorname{Trn}} m_{i j}+q_{i} \leq 1, \quad i \in \operatorname{Trn},
\end{gathered}
$$

$$
\begin{aligned}
& \sum_{i \in \operatorname{Trn}} m_{i j}+q_{j} \leq 1, \quad j \in \operatorname{Trn} \\
& m_{i j}+m_{j i} \leq 1, \quad i, j \in \operatorname{Trn} .
\end{aligned}
$$

(iv) The number of detoured trains cannot exceed the residual capacity of alternative lines:

$$
\sum_{i \in \operatorname{Trn}} o_{i} \leq \Delta C
$$

(v) The minimal number of the trains that being applied rerouting actions:

$$
\sum_{i \in \operatorname{Trn}} \sum_{j \in \operatorname{Trn}} m_{i j}+\sum_{i \in \operatorname{Trn}} q_{i}+\sum_{i \in \operatorname{Trn}} o_{i} \geq \delta .
$$

\subsection{Model of Arrival/Departure Time Adjustment Layer}

4.3.1. The Decision Variables. The decision variable are described as follows:

$s_{p}, e_{p}:$ start time and end time of interval $p$;

$d_{p}$ : delay of interval $p$, which is defined as the difference between the departure time after adjustment and the planned departure time in the original timetable:

$b_{i}= \begin{cases}1, & \text { if train } i \text { reaches its final considered stop with } \\ & \text { a delay larger than } w_{i}, \text { where } i \in \operatorname{Trn} \\ 0, & \text { otherwise, }\end{cases}$ 


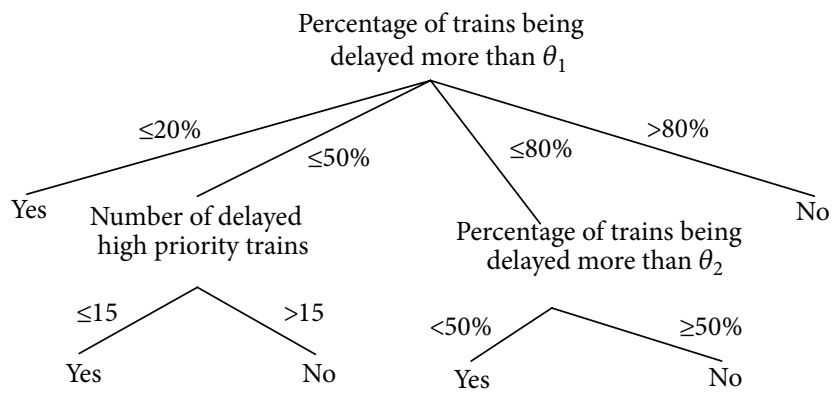

FIgURE 3: Illustration of the smallest decision tree obtained from Table 1.

$\eta_{p l}= \begin{cases}1, & \text { if interval } p \text { uses track } l, \text { where } p \in \operatorname{Itv}_{k} \\ & l \in \operatorname{Trk}_{k} \text { and } k \in \operatorname{Stn} \\ 0, & \text { otherwise, }\end{cases}$

$\alpha_{p \tilde{p}} \begin{cases}1, & \text { if interval } p \text { occurs before } \widetilde{p} \text { as in the } \\ & \text { initial timetable, where } p, \tilde{p} \in \mathrm{Itv}_{k}, k \in \mathrm{Seg} \\ 0, & \text { otherwise, }\end{cases}$

$\beta_{p \tilde{p}}= \begin{cases}1, & \text { if interval } p \text { is changed to occur after } \tilde{p} \\ & \text { where } p, \widetilde{p} \in \operatorname{Itv}_{k}, k \in \text { Seg } \\ 0, & \text { otherwise. }\end{cases}$

\subsubsection{Objective Functions}

(i) To minimize the delay cost,

$$
\operatorname{Minimize} \sum_{i \in \operatorname{Trn}}\left(c_{i}^{\text {delay }} \cdot \sum_{p \in \mathrm{Itv}_{i}^{\mathrm{stn}}} d_{p}\right)
$$

(ii) To minimize the number of trains exceeding delay tolerance,

$$
\text { Minimize } \sum_{i \in \operatorname{Trn}} b_{i}
$$

\subsubsection{Constraints}

(i) Interval restrictions are as follows:

$$
e_{p} \geq s_{p}+z_{p}, \quad p \in \text { Itv. }
$$

The real departure time cannot be earlier than the original departure time:

$$
\begin{gathered}
e_{p} \geq \text { inie }_{p}, \quad p \in \text { Itv }, \quad h_{p}=1, \\
e_{p}-\text { inie }_{p}=d_{p}, \quad p \in \text { Itv. }
\end{gathered}
$$

(ii) Track restrictions

$$
\sum_{l \in \operatorname{Trk}_{k}} \eta_{p l}=1, \quad p \in \operatorname{Itv}_{k}, k \in \operatorname{Stn} .
$$

(iii) Connectivity restrictions are as follows:

$$
e_{p}=s_{p+1}, \quad p \in \operatorname{Itv}_{i}, p \neq \operatorname{last}\left(\operatorname{Itv}_{i}\right), i \in \operatorname{Trn} .
$$

For each station, if two intervals use the same track, at least one of $\alpha$ and $\beta$ is forced to be 1 :

$$
\begin{aligned}
& \eta_{p l}+\eta_{\tilde{p l}}-1 \leq \alpha_{p \tilde{p}}+\beta_{p \tilde{p}}, \\
& p, \tilde{p} \in \operatorname{Itv}_{k}, \quad p<\tilde{p}, \quad l \in \operatorname{Trk}_{k}, \quad k \in \operatorname{Stn} .
\end{aligned}
$$

One train can enter a station after the preceding train, which uses the same track, leaves it:

$$
\begin{array}{ll}
s_{\tilde{p}}-e_{p} \geq f_{k} \alpha_{p \tilde{p}}-M\left(1-\alpha_{p \tilde{p}}\right), & p, \tilde{p} \in \operatorname{Itv}_{k}, k \in \operatorname{Stn} \\
s_{p}-e_{\tilde{p}} \geq f_{k} \beta_{p \tilde{p}}-M\left(1-\beta_{p \tilde{p}}\right), & p, \tilde{p} \in \operatorname{Itv}_{k}, k \in \text { Stn. }
\end{array}
$$

For each section, at least one of $\alpha$ and $\beta$ is forced to be 1 because there is only one track:

$$
\alpha_{p \tilde{p}}+\beta_{p \tilde{p}}=1, \quad p, \tilde{p} \in \operatorname{Itv}_{k}, k \in \operatorname{Sec} .
$$

Headways for each section are as follows:

$$
\begin{array}{ll}
s_{\tilde{p}}-s_{p} \geq h w_{k} \alpha_{p \tilde{p}}-M\left(1-\alpha_{p \tilde{p}}\right), & p, \tilde{p} \in \operatorname{Itv}_{k}, k \in \operatorname{Sec} \\
s_{p}-s_{\tilde{p}} \geq h w_{k} \beta_{p \tilde{p}}-M\left(1-\beta_{p \tilde{p}}\right), & p, \tilde{p} \in \operatorname{Itv}_{k}, k \in \operatorname{Sec} .
\end{array}
$$

(iv) Auxiliary restrictions are as follows:

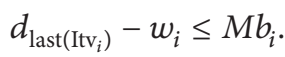

\section{Case Study}

The proposed DSM is simulated on the busiest part of BeijingShanghai high-speed line, between Nanjing (Ning for short) and Shanghai (Hu for short). In the rest of this paper, "HuNing Part" is used to represent this part of the BeijingShanghai high-speed line. As shown in Figure 5, besides Beijing-Shanghai high-speed line, there are two other lines connecting $\mathrm{Hu}$ and Ning, which are Hu-Ning inter-city highspeed line and existing Beijing-Shanghai line. Since all of 
them are double-track lines, we only consider the direction from Ning to $\mathrm{Hu}$ without loss of generality. All the MILP models are solved by IBM ILOG CPLEX 12.2.

As shown in Figure 4, there are seven stations on the Hu-Ning Part, Nanjing South, Zhenjiang West, Changzhou North, Wuxi East, Suzhou North, Kunshan South, and Hongqiao (Shanghai). A specific section is the railway part between two consecutive stations; for example, NanjingZhenjiang Section is the railway part between Nanjing South Station and Zhenjiang West Station that is regarded as the 1st section on the Hu-Ning Part. The lengths of the six sections starting from Nanjing-Zhenjiang Section are $65110 \mathrm{~m}, 61050 \mathrm{~m}, 56400 \mathrm{~m}, 26810 \mathrm{~m}, 31350 \mathrm{~m}$, and $43570 \mathrm{~m}$, respectively. Its daily service starts at 6:30 am and ends at 11:30 pm. The planned timetable is obtained from BeijingShanghai High-speed Line Timetable Planning Lab, Beijing Jiao Tong University. As currently planned, there are 60 trains in the initial timetable. The trains from Beijing-Shanghai line are called self-line trains, while those from Riverside line are called cross-line trains. Fourteen high-speed trains from Beijing-Shanghai line take $L$ and $D J$ as the train number prefix, 38 quasi-high-speed trains from Beijing-Shanghai line take $G$ as the train number, and 8 quasi-high-speed trains from Riverside line take $K$ as the train number. As in reality, self-line trains have higher priority than cross-line trains. As for self-line trains, the high-speed trains have higher priority than the quasi-high speed trains. The costs per time unit delay for the three kinds of trains are set to 5, 3, and 1, respectively. The costs of three kinds of rerouting strategies for trains (canceling train, detouring train, and merging train) are set based on the train's priority and its delay time. Delay tolerance for trains is set to $30 \mathrm{~min}$. The minimum separation time on track possession in each station is set to $1 \mathrm{~min}$.

In Figure 5, the high-speed line, inter-city line, and existing line are represented by $\overline{\mathrm{NH}}, \overline{\mathrm{NH}}_{i c}$, and $\overline{\mathrm{NH}}_{e}$, respectively. Correspondingly, the capacities are $C, C_{i c}$, and $C_{e}$, and the demands are $D, D_{i c}$, and $D_{e}$. The unit of both capacity and demand is the number of trains. Suppose there is a speed restriction on $\overline{\mathrm{NH}}$, resulting in that the capacity of $\overline{\mathrm{NH}}$ reduces to $C^{\prime} . \overline{\mathrm{NH}}_{i c}$ and $\overline{\mathrm{NH}}_{e}$ are in normal service, with known residual capacity $\Delta C_{i c}=C_{i e}-D_{i e}$ and $\Delta C_{e}=C_{e}-$ $D_{e}$, respectively. Before rescheduling the timetable in case of speed restriction, $C^{\prime}$ needs to be evaluated.

Although there are two different train speeds on the Beijing-Shanghai Part in the planned timetable, which are high-speed $(350 \mathrm{~km} / \mathrm{hr})$ and quasi-high speed $(300 \mathrm{~km} / \mathrm{hr})$, the speed of trains will become uniform when the speed restriction is lower than $300 \mathrm{~km} / \mathrm{h}$. Thus, in the two deduction factors mentioned at the end of Line Capacity Evaluation section, only the stopover of trains needs to be considered, but not the meeting of the trains. The deduction factor for single high-speed train with stopovers with regard to high-speed train without stopovers can be obtained by the following equation [24]:

$$
\varepsilon^{\text {high,stop }}=\frac{I+T}{I},
$$

where $I$ denotes the minimum headway between consecutive trains and $T$ denotes the minimum stopping time at stations. Then, the average deduction factor for multiple highspeed trains can be obtained by the equation below [24]:

$$
\varepsilon_{\text {high }}=\alpha^{\text {high }}+\alpha^{\text {high,stop }} \varepsilon^{\text {high,stop }}
$$

where $\alpha^{\text {high }}$ denotes the ratio of high-speed trains without stopovers and $\alpha$ high,stop denotes the ratio of high-speed trains with stopovers. Given equations (1), (23), and (24) the capacity of high-speed rail line with trains of uniformed speed with stopovers can be obtained by the following equation:

$$
C^{\prime}=\frac{n_{\text {max }}}{\varepsilon_{\text {high }}}=\frac{1440-t_{\text {maintenance }}-t_{\text {inefficacy }}}{\varepsilon_{\text {high }} I},
$$

where the headway $I$ is calculated by (Number of blocks * Length of block + Length of Train)/Train's average speed [24].

In the case study, $T$ is 3 minutes. Since the capacity of the sections with the most stopovers restrains the capacity of the whole line, $\alpha^{\text {high }}=16 / 43, \alpha^{\text {high,stop }}=27 / 43$ are calculated in these sections. As planned, $t_{\text {maintenance }}=6$ hours $(0: 00 \mathrm{AM}$ to $6: 00 \mathrm{AM}) . t_{\text {inefficacy }} \approx \mathrm{dis} / v$ is related to the total distance dis $(284 \mathrm{~km}$ in this case) and the restricted train speed $v$. The lower the restricted speed is, the longer the inefficacy will be. The capacity of the Hu-Ning Part in various speed restrictions is calculated by (23)-(25), and the results are shown in Table 3.

In the case study, demand $D=43$. It can be seen from Table 3 that $C^{\prime}$ decreases as the speed restriction gets lower. But it never gets smaller than $D$, which means the demand of Hu-Ning Part can always be fulfilled even when the speed is restricted down to $50 \mathrm{~km} / \mathrm{hr}$. The reason why the demand is so small may be that the obtained timetable is designed for the first step operation of the Beijing-Shanghai line, at which time of the high-speed network in blueprint has not formed yet.

Since $C^{\prime} \geq D$ holds in all the speed restriction cases, rerouting actions will not be taken at the first place as stated in the Rerouting Management section. The arrival/departure time adjustment optimization is executed, and the results are given in Table 4 , where $\eta_{1}$ denotes the number of trains with a delay not less than 30 mins, $\eta_{2}$ denotes the number of trains with a delay not less than 60 mins, $\lambda$ denotes the number of trains entering the last section beyond service hour, and $\gamma$ denotes the number of delayed self-line trains. The values in brackets are the results with no rescheduling.

It can be seen from Table 4 that no train will be delayed for more than 30 mins when the speed restriction takes place in 1st section or 1st and 2nd sections, and the speed limitation is higher than $100 \mathrm{~km} / \mathrm{hr}$. There is a finding that the delay of 1st and 2 nd sections speed restriction is more serious than that of 1st section speed restriction in terms of all the metrics. The results suggest that the longer the restricted distance is or the lower the speed limitation is, the more serious the delay will be. In addition, it can be seen from the results that both the total delay time and the number of delayed trains obtained with the proposed approach are less than those obtained without rescheduling.

As proposed in the Decision Tree-based Result Assessment section, a decision tree is used to evaluate the rescheduled timetable. In the case study, it is assumed that the 


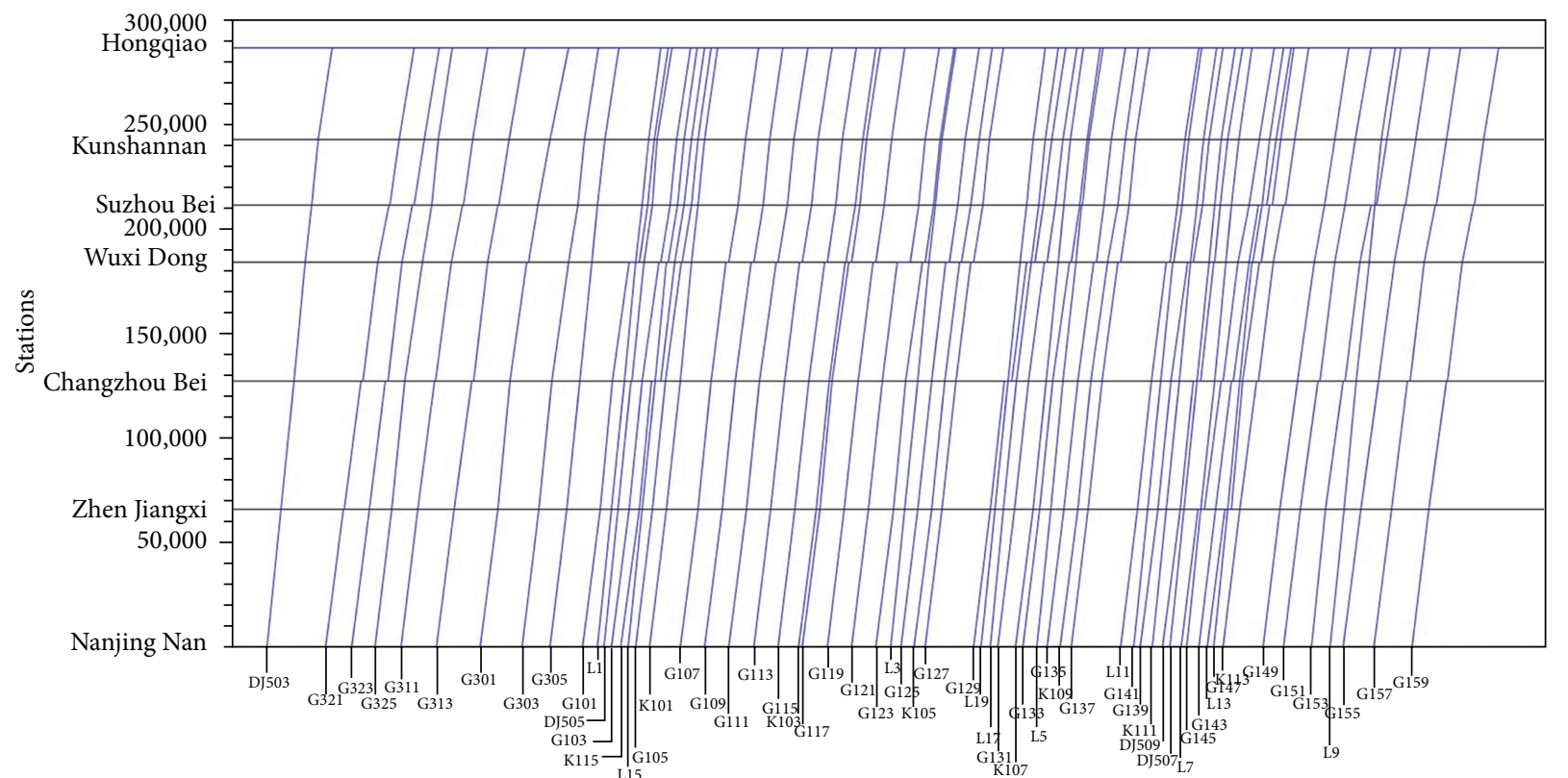

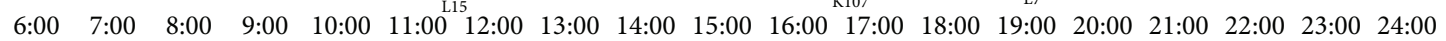

FIgure 4: The planned timetable of Hu-Ning Part.

TABLE 3: Capacity of Hu-Ning part in speed restrictions.

\begin{tabular}{lccccc}
\hline $\begin{array}{l}\text { Restricted speed } \\
(\mathrm{km} / \mathrm{hr})\end{array}$ & $t_{\text {inefficacy }}(\mathrm{hr})$ & $I(\mathrm{~min})$ & $n_{\text {max }}$ & $\varepsilon^{\text {high,stop }}$ & $\mathcal{E}^{\text {high }}$ \\
\hline 300 & N/A & 2.3 & 469 & 2.3 & 1.82 \\
200 & 1 & 2 & 510 & 2.5 & 1.94 \\
100 & 2 & 2.2 & 436 & 2.36 & 1.85 \\
80 & 3 & 2.7 & 333 & 2.1 & 262 \\
50 & 6 & 2.4 & 300 & 2.25 & 1.69 \\
\hline
\end{tabular}

TABLE 4: Evaluation results on the rescheduled timetable.

\begin{tabular}{|c|c|c|c|c|c|c|c|}
\hline Restricted section(s) & Speed limitation $(\mathrm{km} / \mathrm{h})$ & $\eta_{1}$ & $\eta_{2}$ & $\lambda$ & $\gamma$ & Total delay (min) & Calculation time $(\mathrm{s})$ \\
\hline \multirow{6}{*}{ 1st section } & 300 & $0(0)$ & $0(0)$ & $0(0)$ & $1(14)$ & $3.81(23.1)$ & 4.83 \\
\hline & 200 & $0(0)$ & $0(0)$ & $0(0)$ & $25(52)$ & $98.21(259.6)$ & 5.24 \\
\hline & 150 & $0(0)$ & $0(0)$ & $0(0)$ & $47(52)$ & $384.81(810.2)$ & 18.69 \\
\hline & 100 & $0(0)$ & $0(0)$ & $0(0)$ & $52(52)$ & $1158.81(1605.8)$ & 9.22 \\
\hline & 80 & $23(60)$ & $0(0)$ & $0(0)$ & $52(52)$ & $1743.31(2017.6)$ & 6.63 \\
\hline & 50 & $40(0)$ & $20(60)$ & $1(1)$ & $52(52)$ & $3501.31(3775.23)$ & 6.69 \\
\hline \multirow{6}{*}{ 1st and 2nd sections } & 300 & $0(0)$ & $0(0)$ & $0(0)$ & $4(52)$ & $7(41.44)$ & 5.22 \\
\hline & 200 & $0(0)$ & $0(0)$ & $0(0)$ & $47(52)$ & $383.13(508.28)$ & 8.13 \\
\hline & 150 & $0(0)$ & $0(0)$ & $0(0)$ & $52(52)$ & $1125.28(1264.98)$ & 6.19 \\
\hline & 100 & $60(60)$ & $0(0)$ & $0(1)$ & $52(52)$ & $2640.14(2779.05)$ & 5.28 \\
\hline & 80 & $18(0)$ & $42(60)$ & $1(1)$ & $52(52)$ & 3776.15 (3911.99) & 5.81 \\
\hline & 50 & $0(0)$ & $60(60)$ & $3(3)$ & $52(52)$ & $7180.28(7320.87)$ & 5.67 \\
\hline \multirow{6}{*}{ All (1st-6th) sections } & 300 & $0(0)$ & $0(0)$ & $0(0)$ & $15(52)$ & $54.05(93.63)$ & 5.13 \\
\hline & 200 & $11(46)$ & $0(0)$ & $0(0)$ & $52(52)$ & $1359.95(1435.96)$ & 3.7 \\
\hline & 150 & $51(0)$ & $9(60)$ & $0(1)$ & $52(52)$ & 3065.95 (3141.71) & 3.72 \\
\hline & 100 & $0(0)$ & $60(60)$ & $2(3)$ & $52(52)$ & $6476.95(6553.22)$ & 4.27 \\
\hline & 80 & $0(0)$ & $60(60)$ & $4(5)$ & $52(52)$ & 9035.95 (9111.78) & 3.67 \\
\hline & 50 & $0(0)$ & $60(60)$ & $11(15)$ & $52(52)$ & $16716.15(16787.6)$ & 4.14 \\
\hline
\end{tabular}




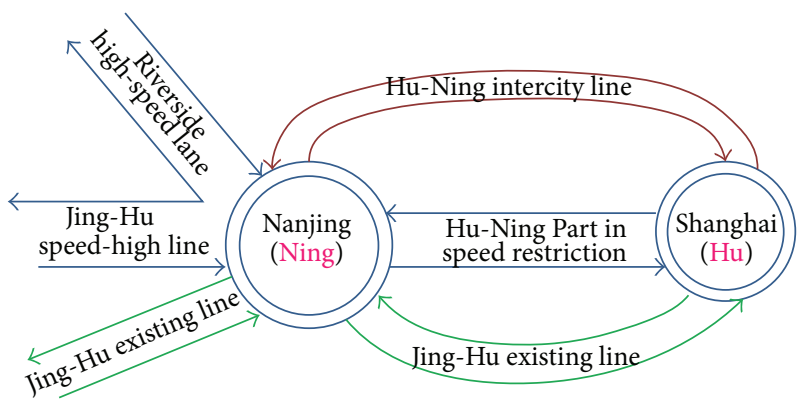

FIgURE 5: The railway network around Nanjing (Ning).

decision tree only comprises of one node, which is $\lambda=0$. In other words, only if all the trains can enter the last section in service hour, the rescheduled result can be accepted. With this single-node decision tree, the unaccepted results are identified in Table 4, including 1st section-50 km/hr, 1st and 2nd sections $-80 \mathrm{~km} / \mathrm{hr}$, 1st and 2nd sections $-50 \mathrm{~km} / \mathrm{hr}$, all sections $-100 \mathrm{~km} / \mathrm{hr}$, all sections $-80 \mathrm{~km} / \mathrm{hr}$, and all sections$50 \mathrm{~km} / \mathrm{hr}$. The results imply that $C^{\prime}$ is not fully utilized.

As proposed in DSM, if a result is not accepted with result assessment, it goes back to the rerouting management module. In the case study, two rerouting strategies are considered, for $C^{\prime} \geq D$. One is to merge/detour/cancel some trains, represented by RS-1. The other one is to advance departure time, represented by RS-2.

(i) RS-1: In the optimization model of the rerouting management layer, we assume residual capacities $\Delta C_{i c}=3$ and $\Delta C_{e}=2$. Advanced departure time is not allowed. Hence, the calculated maximum capacity is not applicable with this strategy. The strategy RS-1 is tested with the minimum rerouted number of trains $\delta$ increasing from 1 to 13 .

(ii) RS-2: The departure time is allowed to be advanced by relaxing the second constraint of interval restrictions in the time adjustment-layer optimization model.

The comparison results are shown in Table 5.

It can be seen from Table 5 that detouring trains strategy has a high priority, merging trains strategy has a medium priority, and canceling trains strategy has a low priority according to the costs, respectively. The K-trains (K101, K103, K105, K107, K109, K111, K113, and K115) and G-trains (G149, G151, G153, G155, G157, and G159) take a detour or are cancelled because that the K-trains are cross-line trains that have lower $c^{\text {detour }}$ and $c^{\text {cancel }}$ than other trains, and the Gtrains that depart from Nanjing South after 8 pm have a lower $c^{\text {detour }}$ and $c^{\text {cancel }}$ than other trains. The detoured train G151 is replaced by L9 when the speed limitation is lower than $80 \mathrm{~km} / \mathrm{h}$, and the speed restriction takes place in all sections. This may be explained that $c^{\text {detour }}$ and $c^{\text {cancel }}$ are related not only with the train priority but also with the speed limitation, and L9 departs after G151 from Nanjing South.

In addition, there is a finding that the results of RS-2 outperform RS-1 in terms of all the metrics. Although the results obtained by using RS-1 are better than those before rerouting management shown in Table 4, the improvement is not big. The difference may be only resulted from the reduction of trains. Consequently, half of the results are still not acceptable based on the decision tree. In contrast, using RS-2 results is significant improvement for all the scenarios. Not only all the rescheduled timetables are acceptable, the values of all the metrics lower down considerably. The comparison result implies that to increase the utilization of line capacity is more helpful than to reduce demand when the train departure is sparse.

\section{Conclusion and Future Work}

This paper proposed a DSM for dispatchers to deal with timetable rescheduling in case of extensive speed restriction. The main contributions include the following. (1) The rerouting optimization is incorporated into the timetable rescheduling with a bilayer optimization model; (2) the line capacity calculation is innovatively used in solving timetable rescheduling problem to guide the selection of rerouting actions and parameters; (3) dispatchers are effectively involved into the iterative timetable rescheduling so that their valuable experience can be leveraged, while most research work on timetable rescheduling focuses on the optimization model itself.

The proposed decomposition into two optimization layers, including rerouting management and arrival/departure time adjustment, makes the timetable rescheduling optimization problem solvable even in a complex setting with an acceptable computation time. In addition, interposition and guidance of dispatchers are introduced into the optimization procedure with the decomposition, resulting in a more practical approach for real-world application. Despite these advantages, the optimality of the original timetable rescheduling optimization problem may be sacrificed in a sense due to the decomposition. The timetable rescheduling usually involves multiple objectives, of which the optimal solutions constitute a Pareto-front. When making the decision on which trains to be rerouted in the rerouting management layer, there is no way of considering the potential delay that each train will induce into the timetable. So it is possible that a good solution is lost with rerouting an improper train, thereby losing the corresponding Pareto-optimal point. Fortunately, the arrival/departure time adjustment layer guarantees to find the global optimal solution. Hence, a Pareto-optimal solution will be found in the end. In summary, the optimality is sacrificed in the sense that some Pareto-optimal solution is missed. This is acceptable because it is hard to say that one objective is more important than another in daily operation.

The proposed mechanism, methods, and models are simulated on the busiest part of the Beijing-Shanghai highspeed rail line. The proposed DSM showed good performance in the sense that the costs of delay and rerouting can be balanced and optimized iteratively. Additionally, a significant finding from the case study is that the most important step is to increase the utilization of the rail line in case of extensive speed restriction. 


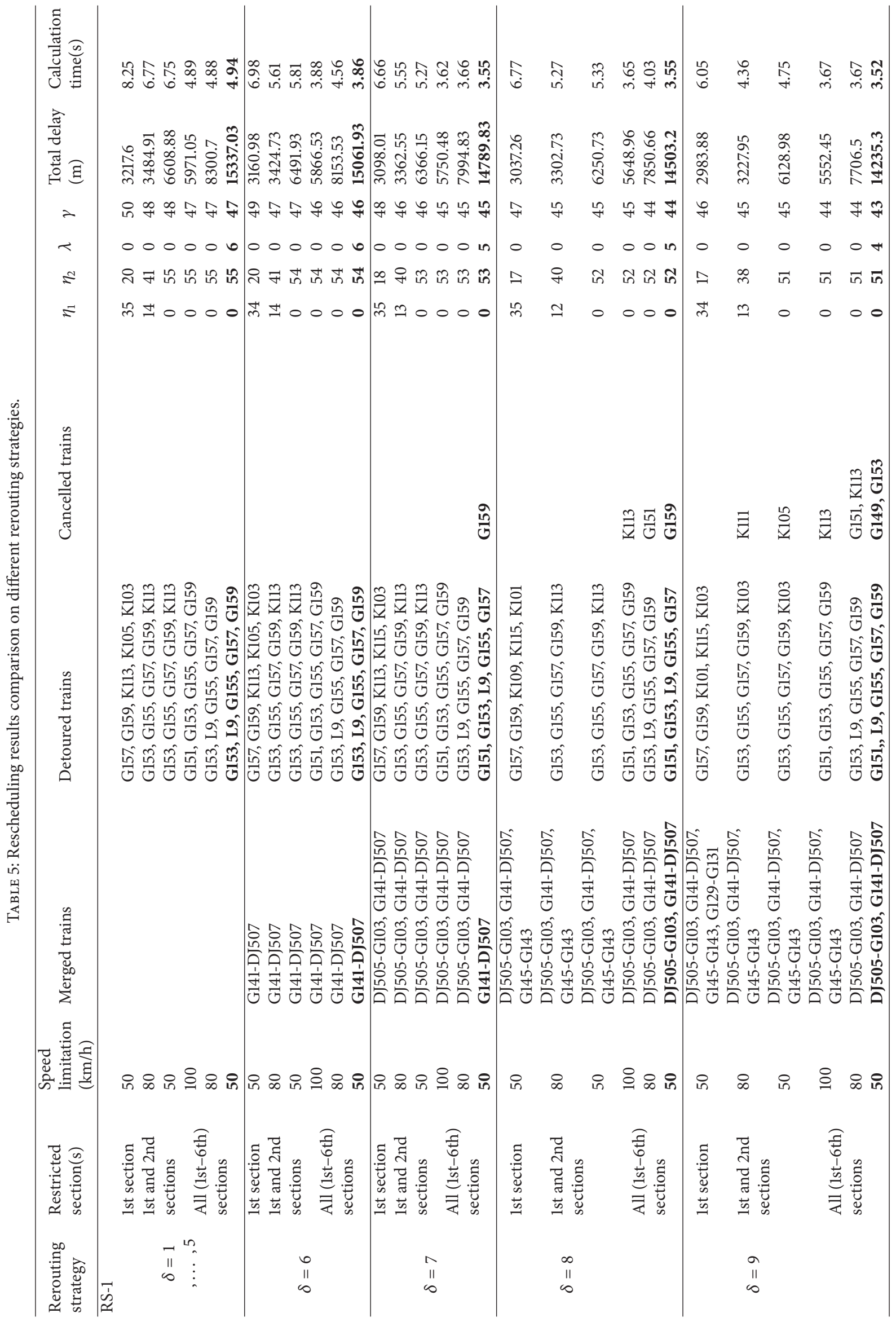




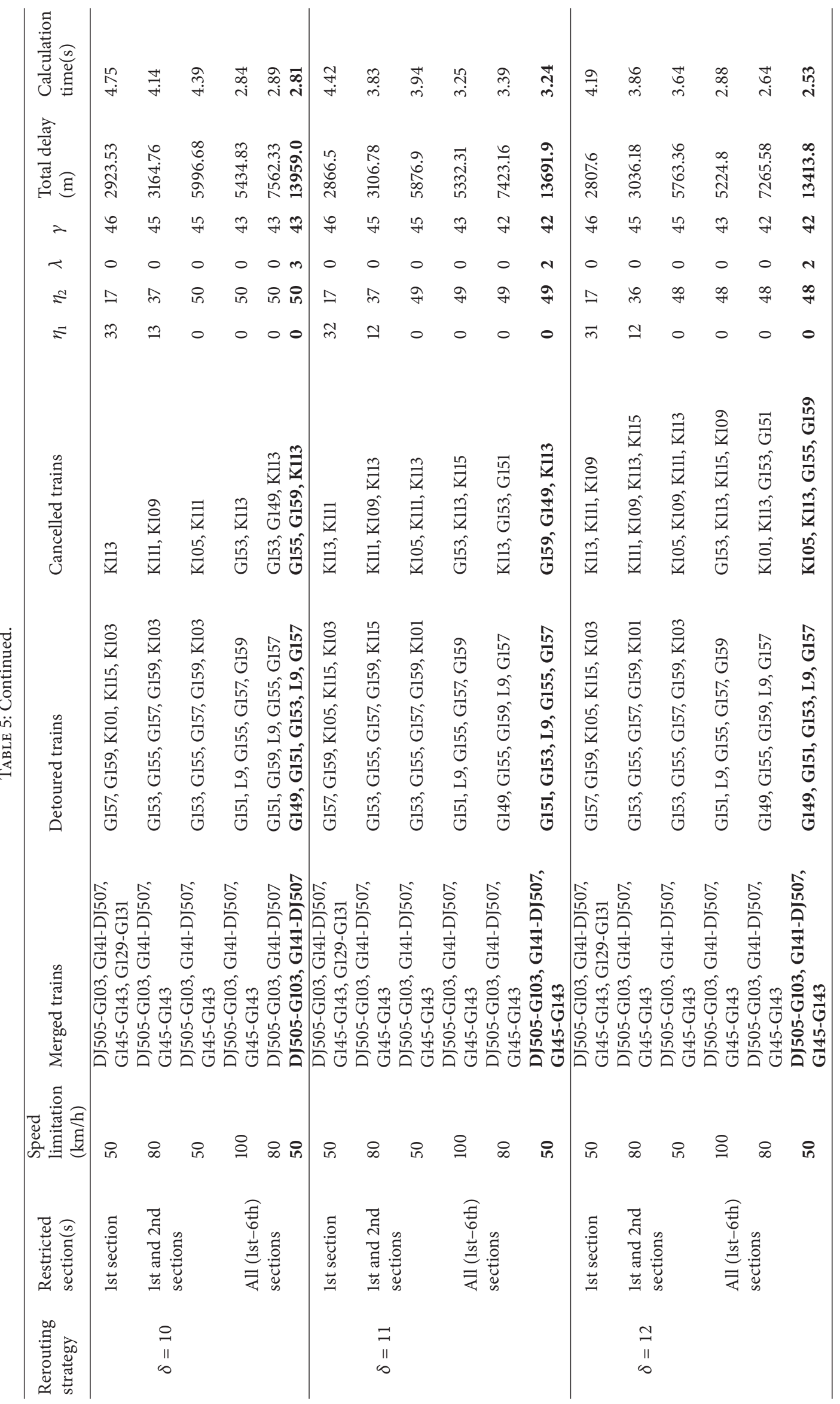




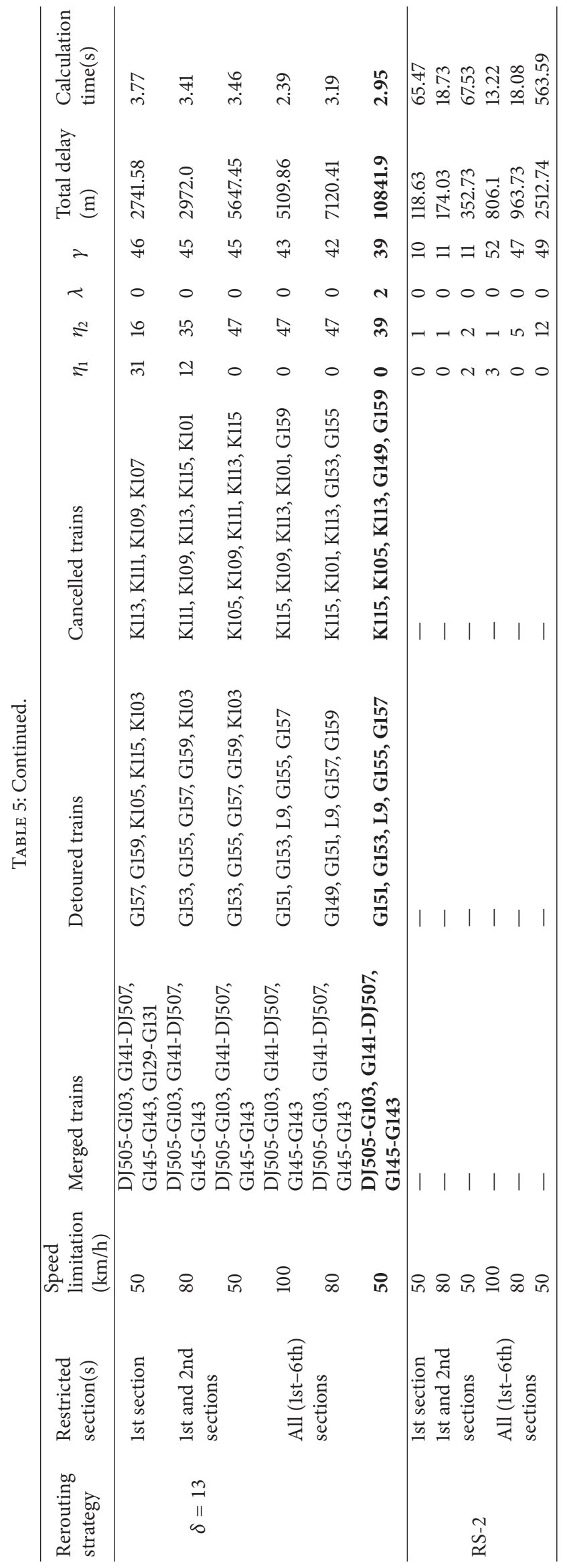


There remain some interesting areas to explore around the proposed DSM. Firstly, the speed restriction is set per day per section; how to make the setting more flexible is an issue. Secondly, the rolling-stock rebalancing is yet to be considered together with the timetable rescheduling. Moreover, the proposed DSM is designed for decision support purpose. Our ultimate goal is to design and develop a real-time decision support framework in the future.

\section{Conflict of Interests}

The authors declare that there is no conflict of interests regarding the publication of this paper.

\section{Acknowledgments}

This work has been supported by National Natural Science Foundation of China (61074151), National Key Technology Research and Development Program (2009BAG12A10), and National High Technology Research and Development Program of China (2012AA112001).

\section{References}

[1] J. Jacobs, "Reducing delays by means of computer-aided "on-the-spot" rescheduling," Computers in Railways IX, WIT, Southampton, UK, vol. 15, pp. 603-612, 2004.

[2] T. Norio, T. Yoshiaki, T. Noriyuki, H. Chikara, and M. Kunimitsu, "Train rescheduling algorithm which minimizes passengers' dissatisfaction," Transactions of Information Processing Society of Japan, vol. 46, no. 2, pp. 26-38, 2005.

[3] A. D’Ariano, D. Pacciarelli, and M. Pranzo, "A branch and bound algorithm for scheduling trains in a railway network," European Journal of Operational Research, vol. 183, no. 2, pp. 643-657, 2007.

[4] A. D'Ariano, F. Corman, D. Pacciarelli, and M. Pranzo, "Reordering and local rerouting strategies to manage train traffic in real time," Transportation Science, vol. 42, no. 4, pp. 405-419, 2008.

[5] A. D’Ariano and T. Albrecht, "Running time re-optimization during real-time timetable perturbations," in Computers in Railways X, J. Allan, C. A. Brebbia, A. F. Rumsey, G. Sciutto, S. Sone, and C. J. Goodman, Eds., pp. 531-540, WIT, Southampton, UK, July 2006.

[6] A. D’Ariano, M. Pranzo, and I. A. Hansen, "Conflict resolution and train speed coordination for solving real-time timetable perturbations," IEEE Transactions on Intelligent Transportation Systems, vol. 8, no. 2, pp. 208-222, 2007.

[7] A. D’Ariano, F. Corman, D. Pacciarelli, and M. Pranzo, "Reordering and local rerouting strategies to manage train traffic in real time," Transportation Science, vol. 42, no. 4, pp. 405-419, 2008.

[8] A. D’Ariano and M. Pranzo, "An advanced real-time train dispatching system for minimizing the propagation of delays in a dispatching area under severe disturbances," Networks and Spatial Economics, vol. 9, no. 1, pp. 63-84, 2009.

[9] L. Wang, L.-M. Jia, Y. Qin, J. Xu, and W.-T. Mo, "A twolayer optimization model for high-speed railway line planning," Journal of Zhejiang University: SCIENCE A, vol. 12, no. 12, pp. 902-912, 2011.
[10] S. Wegele and E. Schnieder, "Dispatching of train operations using genetic algorithms," in Proceedings of the 9th International Conference on Computer-Aided Scheduling of Public Transport (CASPT '04), San Diego, Calif, USA, 2004.

[11] J. Törnquist and J. A. Persson, "Train traffic deviation handling using tabu search and simulated annealing," in Proceedings of the 38th Annual Hawaii International Conference on System Sciences, p. 73, January 2005.

[12] J. Törnquist and J. A. Persson, "N-tracked railway traffic rescheduling during disturbances," Transportation Research Part B, vol. 41, no. 3, pp. 342-362, 2007.

[13] I. Şahin, "Railway traffic control and train scheduling based on inter-train conflict management," Transportation Research Part $B$, vol. 33, no. 7, pp. 511-534, 1999.

[14] L. Wang, Y. Qin, J. Xu, and L. Jia, "A fuzzy optimization model for high-speed railway timetable rescheduling," Discrete Dynamics in Nature and Society, vol. 2012, Article ID 827073, 22 pages, 2012.

[15] B. Adenso-Díaz, M. Oliva González, and P. González-Torre, "On-line timetable re-scheduling in regional train services," Transportation Research Part B, vol. 33, no. 6, pp. 387-398, 1999.

[16] A. Higgins, E. Kozan, and L. Ferreira, "Optimal scheduling of trains on a single line track," Transportation Research Part B, vol. 30, no. 2, pp. 147-158, 1996.

[17] W. Wang, Y. Mao, J. Jin et al., “Driver's various information process and multi-ruled decision-making mechanism: a fundamental of intelligent driving shaping model," International Journal of Computational Intelligence Systems, vol. 4, no. 3, pp. 297-305, 2011.

[18] H. Guo, W. Wang, W. Guo, X. Jiang, and H. Bubb, "Reliability analysis of pedestrian safety crossing in urban traffic environment," Safety Science, vol. 50, no. 4, pp. 968-973, 2012.

[19] E. R. Kraft, "Jam capacity of single track rail lines," Proceedings of the Transportation Research Forum, vol. 23, no. 1, pp. 461-471, 1982.

[20] B. Szpigel, "Optimal train scheduling on a single track railway," Operational Research, vol. 72, pp. 343-352, 1972.

[21] D. Jovanović and P. T. Harker, "Tactical scheduling of rail operations. The SCAN I system," Transportation Science, vol. 25, no. 1, pp. 46-64, 1991.

[22] N. Welch and J. Gussow, "Expansion of Canadian National Railway's line capacity," Interfaces, vol. 16, no. 1, pp. 51-64, 1986.

[23] UIC leaflet 406, Capacity, UIC, Paris, France, 2004.

[24] H. Yang, Theory of Railway Transportation Planning, China Railway Publishing House, 1999. 


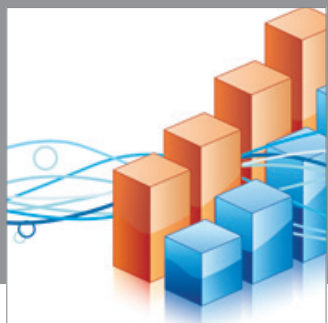

Advances in

Operations Research

mansans

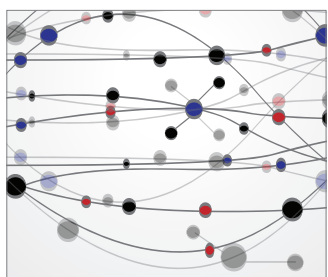

The Scientific World Journal
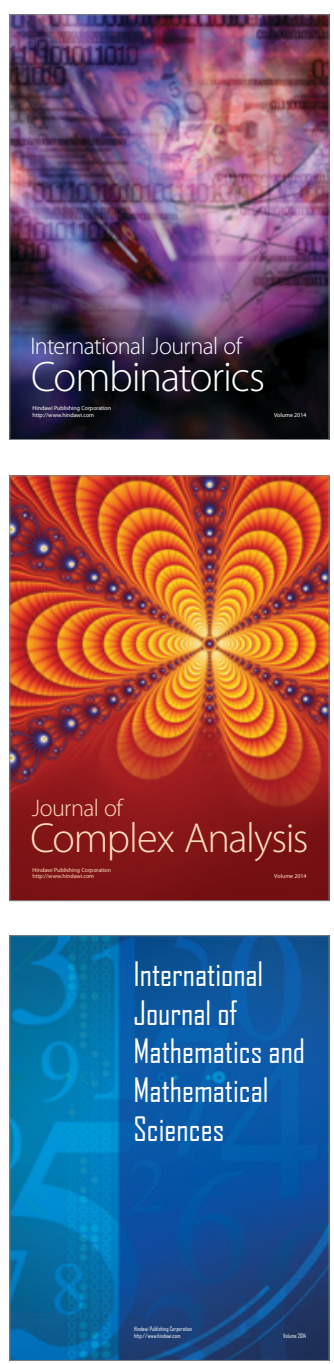
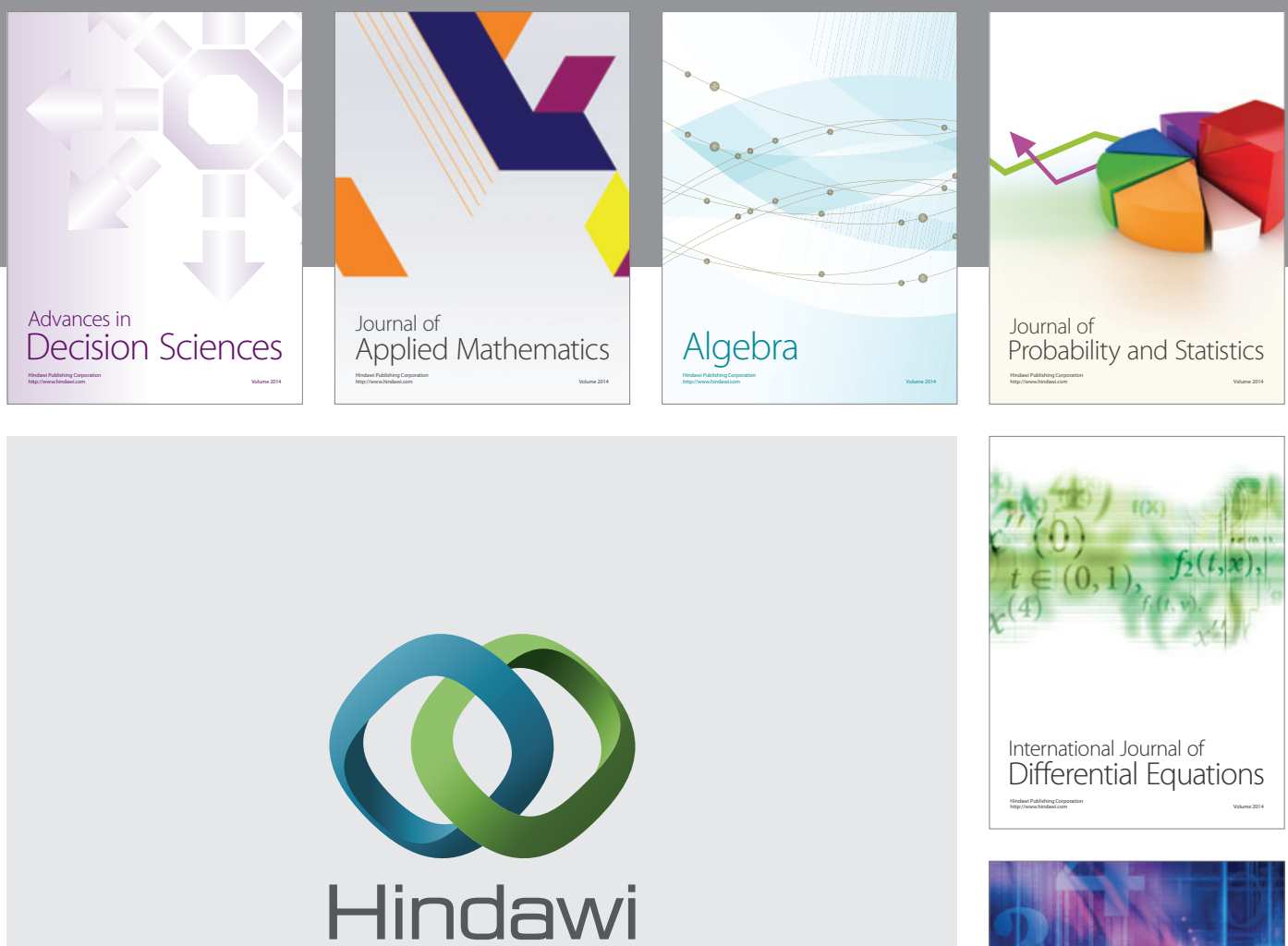

Submit your manuscripts at http://www.hindawi.com
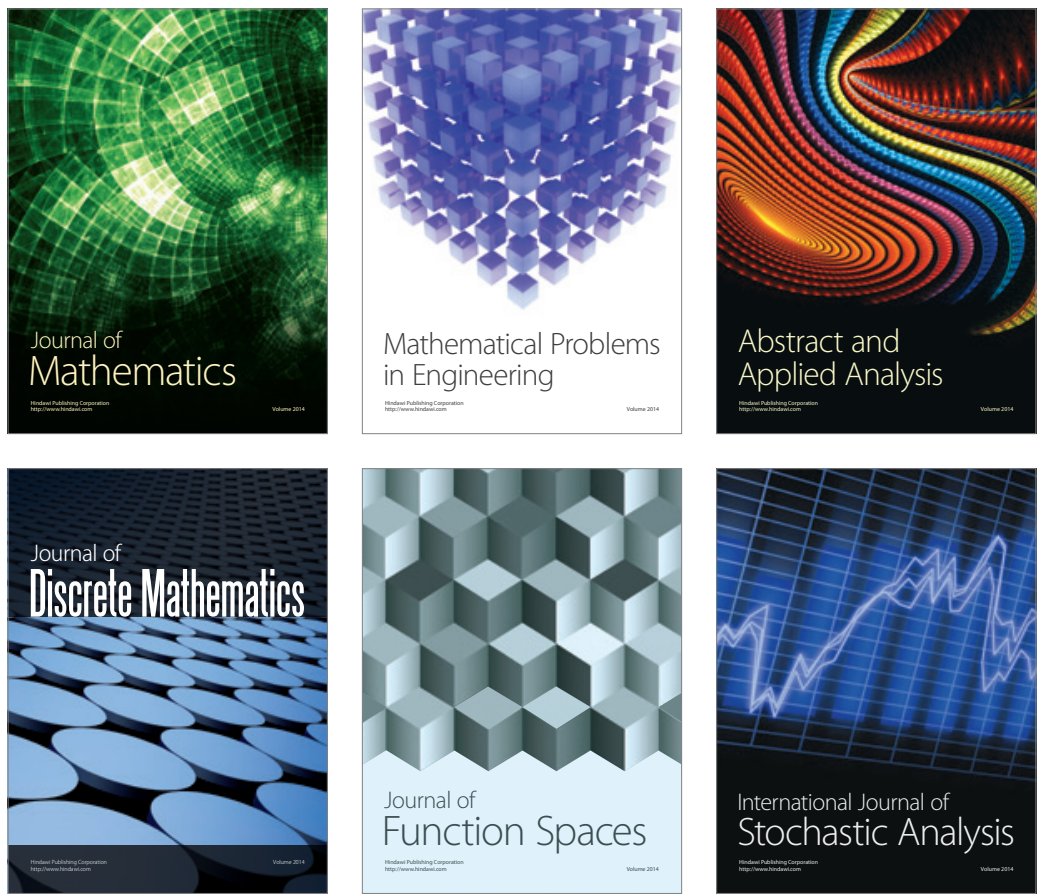

Journal of

Function Spaces

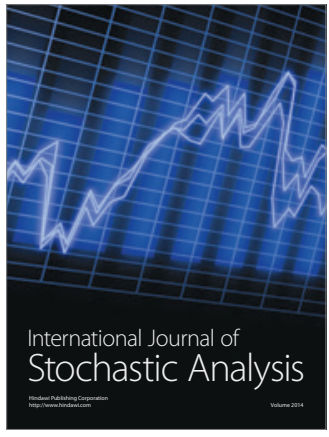

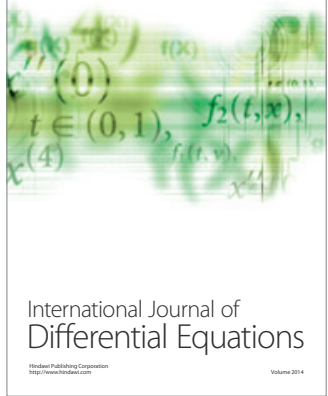
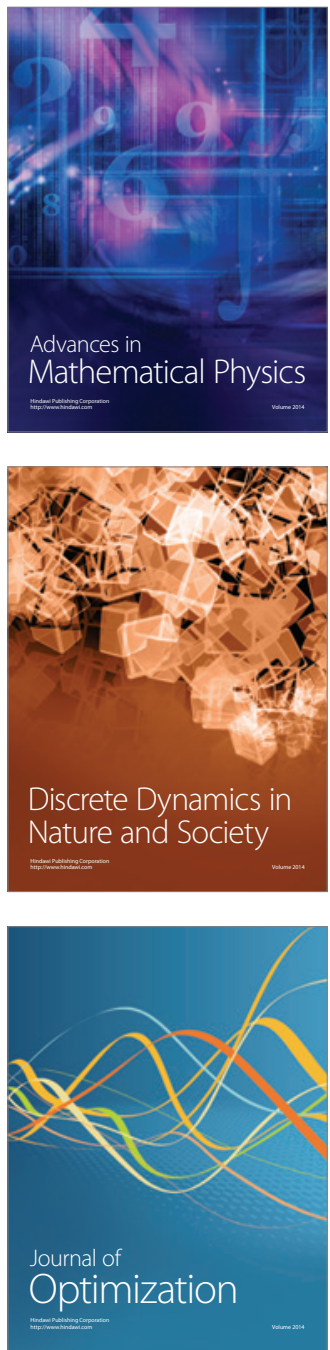